Title : will be set by the publisher

Editors : will be set by the publisher

EAS Publications Series, Vol. ?, 2021

\title{
A NEW TYPE OF SINGULARITY THEOREM
}

\author{
José M M Senovilla ${ }^{1}$
}

\begin{abstract}
A new type of singularity theorem, based on spatial averages of physical quantities, is presented and discussed. Alternatively, the results inform us of when a spacetime can be singularity-free. This theorem provides a decisive observational difference between singular and non-singular, globally hyperbolic, open cosmological models.
\end{abstract}

\section{Introduction}

In 1990 I presented the following line-element (Senovilla 1990)

$$
\begin{gathered}
d s^{2}=\cosh ^{4}(a t) \cosh ^{2}(3 a \rho)\left(-d t^{2}+d \rho^{2}\right)+ \\
\frac{1}{9 a^{2}} \cosh ^{4}(a t) \cosh ^{-2 / 3}(3 a \rho) \sinh ^{2}(3 a \rho) d \varphi^{2}+\cosh ^{-2}(a t) \cosh ^{-2 / 3}(3 a \rho) d z^{2},
\end{gathered}
$$

given in cylindrical coordinates $\{t, \rho, \varphi, z\}, a>0$ is a constant. It is a cylindrically symmetric perfect-fluid solution of the Einstein field equations. The energy density $\varrho$ and the unit velocity vector field $\vec{u}$ of the fluid are given by

$$
\varrho=15 a^{2} \cosh ^{-4}(a t) \cosh ^{-4}(3 a \rho), \quad \vec{u}=\cosh ^{-2}(a t) \cosh ^{-1}(3 a \rho) \partial_{t}
$$

while the acceleration one-form is non-zero $\boldsymbol{a}=3 a \tanh (3 a \rho) d \rho$. The fluid satisfies the barotropic equation of state ( $p$ is the isotropic pressure)

$$
p=\frac{1}{3} \varrho
$$

which is realistic for radiation-dominated matter, that is, for the initial stages of the present expanding era of the Universe.

The space-time (1.1) satisfies the strongest causality condition, global hyperbolicity, as any $t=$ const. slice is a Cauchy hypersurface. Observe that $\varrho$ and $p$ are regular and positive everywhere. Actually, the space-time (1.1) is completely free

\footnotetext{
${ }^{1}$ Física Teórica, Universidad del País Vasco, Apartado 644, 48080 Bilbao, Spain
} 
Title : will be set by the publisher

of singularities and geodesically complete. For thorough discussions see (Chinea et al:1992, Ruiz and Senovilla 1992, Senovilla 1998a sect. 7.6). Given that $p>0$ the solution appears to be "cosmological", as it cannot be matched to any vacuum exterior and matter is present everywhere. The fluid expansion reads

$$
\theta=\nabla_{\mu} u^{\mu}=3 a \frac{\sinh (a t)}{\cosh ^{3}(a t) \cosh (3 a \rho)}
$$

so that the entire universe is contracting everywhere if $t<0$ and expanding if $t>0$, having a rebound at $t=0$. Thus, this simple model showed that there exist wellbehaved classical models expanding everywhere, satisfying all energy and causality conditions, and singularity-free. However, (1.1) is an unrealistic model to describe the actual Universe because the cylindrical symmetry implies a type of anisotropy incompatible with observations (Senovilla 1996).

The following questions spring to mind: how many well-founded non-singular solutions are there? did the universe have the choice of a non-singular start of the expansion epoch? As a matter of fact Garfinkle \& Harris (1997) answered the related question of how the Ricci tensor behaves in stationary globally hyperbolic geodesically complete spacetimes. However, the interesting case is non-stationary, of course. This is the case treated in (Senovilla 2007) upon which this contribution is based. The following is a brief summary of the results.

\section{Averaging}

In 1998, Raychaudhuri (1998a) proved that the solution (1.1), as well as the many other singularity-free solutions found since 1990 - see (Senovilla 1998a, 2007) for lists and a review-, has vanishing space-time averages of the energy density and pressure. However, it was soon realized (Saa 1998, Senovilla 1998b) that this same property is shared by all Robertson-Walker models. Hence, these averages do not distinguish between singular and non-singular cosmological models.

Nevertheless, Raychaudhuri was pointing into a very interesting direction: averaging. The use of pure spatial averages (at a given instant of time) occurred to me immediately, and a conjecture was put forward in (Senovilla 1998b). Af-

ter a hazardous life, see e.g. the chronologically key references (Raychaudhuri 1998b, 2000, 2004, 2005, Fernández-Jambrina \& González-Romero 2002, 2004, Fernández-Jambrina 2005), the conjecture was made precise and recently proven in (Senovilla 2007, Jerjen 2007). To understand its rigorous formulation we need to learn a little about spatial averages. 


\subsection{Spatial averages}

Let $\Sigma$ be any spacelike hypersurface and $\boldsymbol{\eta}_{\Sigma}$ its canonical volume element 3-form. The average $\langle f\rangle_{S}$ of any scalar $f$ on a finite portion $S$ of $\Sigma$ is defined by

$$
\langle f\rangle_{S} \equiv \frac{\int_{S} f \boldsymbol{\eta}_{\Sigma}}{\int_{S} \boldsymbol{\eta}_{\Sigma}}=[\operatorname{Vol}(S)]^{-1} \int_{S} f \boldsymbol{\eta}_{\Sigma}
$$

where $\operatorname{Vol}(S)$ is the volume of $S \subseteq \Sigma$. Let $\left\{S_{i}\right\}$ be a continuous sequence of nested such portions converging to $\Sigma$ - for the notion of convergence, see (Jerjen 2007). The spatial average on the whole $\Sigma$ is defined then as:

$$
\langle f\rangle_{\Sigma} \equiv \lim _{i \rightarrow \infty}\left[\operatorname{Vol}\left(S_{i}\right)\right]^{-1} \int_{S_{i}} f \boldsymbol{\eta}_{\Sigma}
$$

This definition is independent of the chosen sequence $\left\{S_{i}\right\}$ (Jerjen 2007).

The averages (2.1) satisfy many useful properties (Senovilla 2007), but the crucial one is that if $f \geq 0$ on $\Sigma$, then $\langle f\rangle_{S} \geq 0$ and the equality requires necessarily that $f \rightarrow 0$ along almost every direction "approaching the boundary" (i.e., when going to infinity). Conversely, if $f>0, f$ is bounded on $\Sigma, f$ is asymptotically nonoscillatory (Jerjen 2007), and $f$ is bounded from below by a positive constant at most along a set of directions of zero measure, then $\langle f\rangle_{S}=0$. The non-oscillatory behaviour introduced in (Jerjen 2007) to correct the proofs in (Senovilla 2007) is perhaps too strong a requirement, but I have not been able to find a milder restriction hitherto.

\section{The Main Theorem}

The conjecture put forward in (Senovilla 1998b) was promoted to an actual singularity theorem in (Senovilla 2007). There were some technical details, however, which made the proof incomplete. These have been corrected in (Jerjen 2007) while keeping the spirit and the body of the original demonstration.

The main theorem reads:

\section{Theorem 1 Assume that}

1. the spacetime contains a non-compact Cauchy hypersurface $\Sigma$ such that its second fundamental form has a trace positive everywhere and asymptotically non-oscillatory

2. $R_{\rho \nu} v^{\rho} v^{\nu} \geq 0$, where $\vec{v}$ is the geodesic vector field orthogonal to $\Sigma$ on $\Sigma$.

3. the scalar curvature $\bar{R}$ of $\Sigma$ is non-positive on average on $\Sigma:\langle\bar{R}\rangle_{\Sigma} \leq 0$;

4. the cosmological constant is non-negative $\Lambda \geq 0$; and

5. the dominant energy condition holds. 
If any single one of the following spatial averages

$$
\begin{aligned}
& \Lambda,\langle\theta\rangle_{\Sigma},\langle\vartheta\rangle_{\Sigma},\left\langle\theta^{2}\right\rangle_{\Sigma},\left\langle\vartheta^{2}\right\rangle_{\Sigma},\left\langle K_{\mu \nu} K^{\mu \nu}\right\rangle_{\Sigma},\langle\bar{R}\rangle_{\Sigma}, \\
&\left\langle v^{\mu} \nabla_{\mu} \vartheta\right\rangle_{\Sigma},\left\langle u^{\mu} \nabla_{\mu} \theta-\nabla_{\mu} a^{\mu}\right\rangle_{\Sigma}, \\
&\left\langle T_{\mu \nu} e_{\alpha}^{\mu} e_{\beta}^{\nu}\right\rangle_{\Sigma}, \quad\left\langle R_{\mu \nu} e_{\alpha}^{\mu} e_{\beta}^{\nu}\right\rangle_{\Sigma} \quad \forall \alpha, \beta=0,1,2,3
\end{aligned}
$$

does not vanish, then all past-directed timelike geodesics are incomplete.

Here $R_{\mu \nu}$ is the Ricci tensor, $\vartheta=\nabla_{\mu} v^{\mu}$ is the expansion of $\vec{v}$, while $\vec{u}$ is any unit timelike vector field such that $\left.\vec{u}\right|_{\Sigma}=\left.\vec{v}\right|_{\Sigma}, \theta$ is its expansion and $\vec{a}=\nabla_{\vec{u}} \vec{u}$ its acceleration vector field. $\left\{\vec{e}_{\alpha}\right\}$ is any orthonormal basis. Finally, $K_{\mu \nu}$ is the second fundamental form of $\Sigma$, which coincides on $\Sigma$ with the shear tensor of $\vec{v}$ (and equals the shear tensor of $\vec{u}$ minus the symmetrized tensor product of $\vec{a}$ and $\vec{u}$.) Of course, $\vartheta=K^{\mu}{ }_{\mu}$ so that $\vec{v}$ is expanding everywhere on $\Sigma$, and the expansion is asymptotically non-oscillatory on $\Sigma$.

Let me briefly comment on the reasonability of the assumptions and the strength and meaning of the derived result. First of all one requires the spacetime to be globally hyperbolic, so that it is causally well-behaved. Furthermore, the Universe is assumed to be open ( $\Sigma$ is non-compact) and everywhere expanding at a given instant of time (say "now"), described by the hypersurface $\Sigma$. The timelike convergence condition must also hold along the geodesic congruence orthogonal to $\Sigma$, and the dominant energy condition is added. All this is standard and well motivated and founded.

On the other hand, the space of the Universe (represented by $\Sigma$ at the expanding instant) is assumed to be non-positively curved on average, while $\Lambda$ is taken as non-positive. Observe that the traditional case with $\Lambda=0$ is included. Both of these requirements are in agreement with present observational data, e.g. the recent data from WMAP. The assumption on $\bar{R}$ still allows, actually, for an everywhere positively curved $\Sigma$. As a matter of fact, the solution (1.1) has $\bar{R}>0$ everywhere (Senovilla 2007).

Given the above, the conclusion of the theorem can be stated in two alternative, but equivalent, ways: (i) in order to have past geodesic completeness, all the displayed spatial averages must vanish, including in particular those of the energy density, the pressure, and all physical components of the energy-momentum tensor; alternatively, (ii) if any of these averages is non-zero, the Universe must be totally past geodesically incomplete. The universality of the past incompleteness is a direct consequence of the technical assumption that $\vartheta$ is asymptotically nonoscillatory, which implies a kind of uniformity for the limit when going to infinity along $\Sigma$ (Jerjen 2007).

The proof of the theorem has three fundamental ingredients: the Raychaudhuri (1955, 1957) equation — see also (Hawking \& Ellis 1973. Wald 1984) - , the simplest standard singularity theorem (Hawking \& Ellis 1973, Penrose 1972, Senovilla 1998a), and the so-called Hamiltonian constraint, e.g. section 10.2 in (Wald 1984). For details, check (Senovilla 2007; Jerjen 2007). 


\section{Conclusions}

The theorem has the following fundamental implication: under the stated assumptions, a non-vanishing average of any component of the energy-momentum tensor, or of any kinematical variable, leads to the existence of a kind of big-bang singularity in the past. Observe that there is no need to assume any specific type of matter content (such as a perfect fluid, scalar field, etc.), as only the physically compelling dominant energy condition is required. This is remarkable.

The theorem concerns "open" models, as the Cauchy hypersurfaces are noncompact. This is, however, not a relevant limitation because there are stronger results proving the geodesic incompleteness of closed models (Hawking \& Ellis 1973. Hawking \& Penrose 1970; Penrose 1965, 1972, Senovilla 1998a). As a matter of fact, closed expanding non-singular models require the violation of the timelike convergence condition $R_{\mu \nu} v^{\mu} v^{\nu} \geq 0$ (Senovilla 1998a) -without this condition, but satisfying the dominant energy condition, there are some non-singular acceptable examples (Mars \& Senovilla 1997, Senovilla1998a).

I would like to stress that the conclusion in theorem 1 is very strong: it tells us that the incompleteness is to the past, and for the entire class of timelike geodesics. Besides, I believe that one can in fact do better and get a stronger theorem such that the non-oscillatory condition on the expansion can be substantially relaxed, or even removed.

Our theorem states, in fact, that a clear, decisive, difference between singular and regular, globally hyperbolic, everywhere expanding (with non-oscillatory behaviour at infinity) cosmological models is that the former can have non-vanishing spatial averages of some of the matter variables, a property which is forbidden for the latter. This may seem to imply that regular globally hyperbolic expanding models cannot be of cosmological type and realistic, as long as the Universe contains a sufficiently homogenous distribution of matter - e.g. not decaying away from us.

On the whole, this is a very satisfactory conclusion.

\section{Acknowledgments}

Comments from Harald Jerjen are acknowledged. Support under grants FIS200401626 (MEC) and GIU06/37 of the University of the Basque Country (UPV/EHU) is gratefully acknowledged.

\section{References}

Chinea F.J., Fernández-Jambrina L. \& Senovilla J.M.M. 1992 Phys. Rev. D 45, 481

Fernández-Jambrina L. 2005, Gen. Rel. Grav. 37, 421

Fernández-Jambrina L. \& González-Romero L.M. 2002, Phys. Rev. D 66, 024027

Fernández-Jambrina L. \& González-Romero L.M. 2004, J. Math. Phys. 45, 2113

Garfinkle D \& Harris S.G. 1997, Class. Quantum Grav. 14139 
Hawking S.W. \& Ellis G.F.R. 1973, The large scale structure of spacetime (Cambridge Univ. Press: Cambridge)

Hawking S.W. \& Penrose R. 1970, Proc. Roy. Soc. London A 314, 529

Jerjen H. 2007, Cosmological singularity theorems and spatial averages, Diploma thesis (University of Neuchâtel, Switzerland). Available online: http://www.unine.ch/phys/string/JerjenThesis.pdf

Mars M. \& Senovilla J.M.M. 1997, Class. Quantum Grav. 14, 205

Penrose R. 1965, Phys. Rev. Lett. 14, 57

Penrose R. 1972, Techniques of Differential Topology in Relativity, Regional Conference Series in Applied Math. 7 (SIAM: Philadelphia)

Raychaudhuri A.K. 1955, Phys. Rev. 98, 1123

Raychaudhuri A.K. 1957, Phys. Rev. 106, 172

Raychaudhuri A.K. 1998a, Phys. Rev. Lett. 80, 654

Raychaudhuri A.K. 1998b, Phys. Rev. Lett. 81, 5033

Raychaudhuri A.K. 2000, Mod. Phys. Lett. A 15, 391

Raychaudhuri A.K. 2004, Gen. Rel. Grav. 36, 343

Raychaudhuri A.K. 2005, Gen. Rel. Grav. 37, 425

Ruiz E. \& Senovilla J.M.M. 1992, Phys. Rev. D 45, 1995

Saa A. 1998, Phys. Rev. Lett. 81, 5031

Senovilla J.M.M. 1990, Phys. Rev. Lett. 64, 2219

Senovilla J.M.M. 1996, Phys. Rev. D 53, 1799

Senovilla J.M.M. 1998a, Gen. Rel. Grav. 30701

Senovilla J.M.M. 1998b, Phys. Rev. Lett. 81, 5032

Senovilla J.M.M. 2007, Pramana (special issue: "Raychaudhuri Equation at the crossroads") 6731

Wald R.M. 1984, General Relativity (The University of Chicago Press: Chicago). 